\title{
Simulation of spheromak evolution and energy confinement ${ }^{a)}$
}

\author{
B. I. Cohen, ${ }^{\text {b) }}$ E. B. Hooper, R. H. Cohen, D. N. Hill, H. S. McLean, \\ R. D. Wood, and S. Woodruff \\ University of California, Lawrence Livermore National Laboratory, Livermore, California 94550 \\ C. R. Sovinec and G. A. Cone \\ Department of Engineering Physics, University of Wisconsin-Madison, Madison, Wisconsin 53706-1609
}

(Received 12 November 2004; accepted 18 January 2005; published online 7 April 2005)

\begin{abstract}
Simulation results are presented that illustrate the formation and decay of a spheromak plasma driven by a coaxial electrostatic plasma gun, and model the plasma energy confinement. The physics of magnetic reconnection during formation is also illuminated. The simulations are performed with the three-dimensional, time-dependent, resistive magnetohydrodynamic NIMROD code [C. R. Sovinec, A. H. Glasser, T. A. Gianakon, D. C. Barnes, R. A. Nebel, S. E. Kruger, D. D. Schnack, S. J. Plimpton, A. Tarditi, and M. S. Chu, J. Comput. Phys. 195, 355 (2004)]. The simulation results are compared to data from the Sustained Spheromak Physics Experiment (SSPX) [E. B. Hooper, L. D. Pearlstein, and R. H. Bulmer, Nucl. Fusion 39, 863 (1999)]. The simulation results are tracking SSPX with increasing fidelity (e.g., improved agreement with measured magnetic fields, fluctuation amplitudes, and electron temperature) as the simulation has been improved in its representations of the experimental geometry, the magnetic bias coils, and the detailed time dependence of the current source driving the plasma gun, and uses realistic parameters. The simulations confirm that controlling the magnetic fluctuations is influenced by the current drive history and by matching the gun current in sustainment approximately to the value corresponding to the eigenvalue in the flux-conserver for the parallel current in a force-free equilibrium. (C) 2005 American Institute of Physics. [DOI: 10.1063/1.1869501]
\end{abstract}

\section{INTRODUCTION}

Electron temperatures near $400 \mathrm{eV}$ were observed transiently in the Los Alamos Compact Torus Experiment (CTX) experiment, ${ }^{1}$ and temperatures of $100-200 \mathrm{eV}$ have been observed in the Livermore Sustained Spheromak Physics Experiment (SSPX). ${ }^{2}$ Understanding the energy confinement in these experiments is a challenging problem. Results from numerical simulations with the NIMROD nonlinear resistive magnetohydrodynamic (MHD) code (at zero or finite plasma pressure) have shown that closed flux surfaces with net current can arise only after electrostatic drive is reduced., 3 Computations in the last year have investigated the importance of inductive effects on energy confinement including the evolution of the temperature and number density using thermal transport coefficients, electrical resistivity, and Ohmic heating. The simulation model is a reasonable approximation because the collisional mean-free path is less than the flux-conserver radius $R=0.5 \mathrm{~m}$ for nominal SSPX plasma parameters with $n \sim 5 \times 10^{19} \mathrm{~m}^{-3}, T \leqslant 35 \mathrm{eV}$, and singly charged ions.

The NIMROD simulations solve nonlinear timedependent equations for particle number density $\left(n_{i}=n_{e}=n\right.$ with quasineutrality), plasma flow velocity $\mathbf{V}$, temperature (assuming $T_{\mathrm{i}}=T_{\mathrm{e}}=T$ ), and magnetic field $\mathbf{B}$. In mks units, the evolution equations are

\footnotetext{
a) Paper PI1B 3, Bull Am. Phys. Soc. 49, 286 (2004).

${ }^{b)}$ Invited speaker.
}

$$
\begin{aligned}
& \frac{\partial n}{\partial t}+\nabla \cdot(n \mathbf{V})=\boldsymbol{\nabla} \cdot D \boldsymbol{\nabla} n, \\
& \begin{array}{l}
\rho\left(\frac{\partial \mathbf{V}}{\partial t}+\mathbf{V} \cdot \nabla \mathbf{V}\right)=\mathbf{J} \times \mathbf{B}-\boldsymbol{\nabla} \boldsymbol{p}+\boldsymbol{\nabla} \cdot \rho \nu \boldsymbol{\nabla} \mathbf{V}, \\
\frac{n k_{B}}{\gamma-1}\left(\frac{\partial T}{\partial t}+\mathbf{V} \cdot \boldsymbol{\nabla} \mathbf{T}\right)=-\frac{p}{2} \boldsymbol{\nabla} \cdot \mathbf{V}+\boldsymbol{\nabla} \cdot n k_{B}\left[\chi_{\|} \hat{\mathbf{b}} \hat{\mathbf{b}}\right.
\end{array} \\
& \left.\quad+\chi_{\perp}(\mathbf{I}-\hat{\mathbf{b}} \hat{\mathbf{b}})\right] \cdot \nabla T+\frac{\eta \mathbf{J}^{2}}{2}, \\
& \frac{\partial \mathbf{B}}{\partial t}=\boldsymbol{\nabla} \times(\mathbf{V} \times \mathbf{B}-\eta \mathbf{J}), \\
& \mu_{0} \mathbf{J}=\boldsymbol{\nabla} \times \mathbf{B},
\end{aligned}
$$

where $p=2 n k_{B} T$ is the sum of electron and ion pressures, and $\hat{\mathbf{b}} \equiv \mathbf{B} /|\mathbf{B}|$ is the evolving magnetic direction vector field. The simulations consider $n, T, \mathbf{V}, \mathbf{B}$, and $\mathbf{J}$ to be functions of all three spatial dimensions and time; thus magnetic fluctuations and anisotropic heat flow are modeled explicitly. The boundary conditions are $\mathbf{E} \times \hat{\mathbf{n}}=\delta \mathbf{B} \cdot \hat{\mathbf{n}}=\mathbf{v}=\boldsymbol{\nabla} \boldsymbol{n} \cdot \hat{\mathbf{n}}=T=0$ on the conducting surfaces. The vacuum magnetic fields from coils are assumed to have soaked through the bounding surfaces. The computational grid has been constructed to conform with the conducting surfaces bounding the domain of the plasma gun and flux conserver. The simulations use bicubic finite elements to represent the poloidal variations (here 
(a)

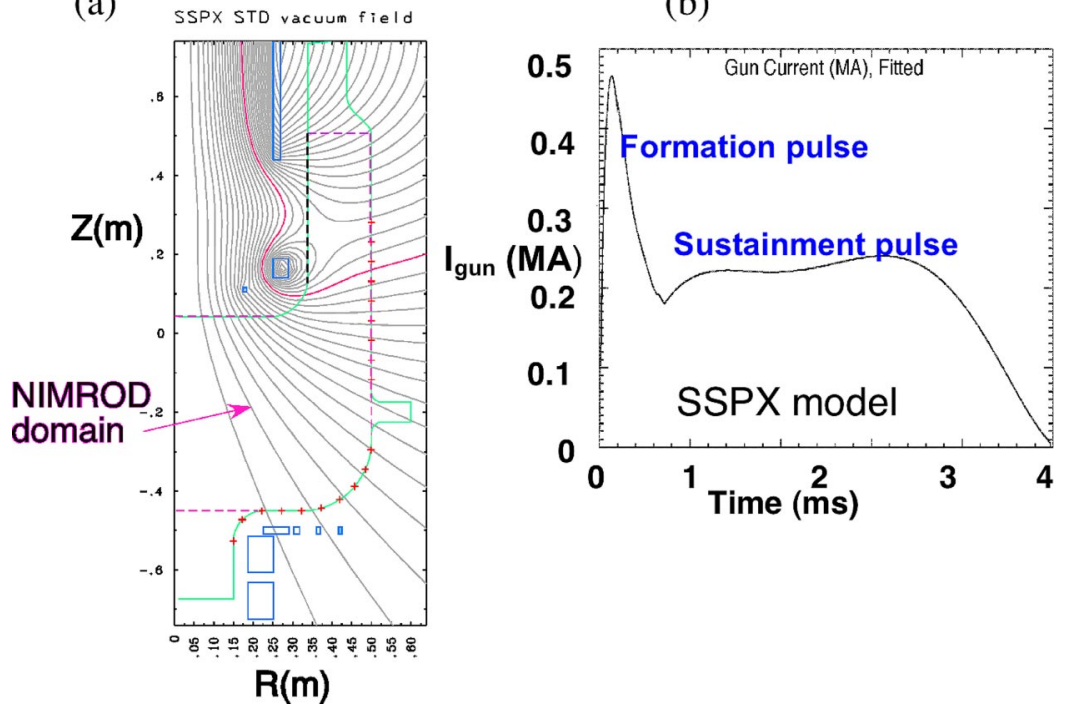

FIG. 1. (a) Vacuum magnetic fields and spheromak configuration. (b) Injector current time history in simulation lam07. we use 24 elements in the direction normal to the electrodes and 32-48 elements parallel to the electrodes) and six toroidal Fourier modes $(0 \leqslant n \leqslant 5)$.

The parallel and perpendicular thermal diffusivities are $\chi_{\|}=387 T^{5 / 2} \mathrm{~m}^{2} / \mathrm{s}$ and $\chi_{\perp}=0.50 T^{-1 / 2} B^{-2} \mathrm{~m}^{2} / \mathrm{s}$ ( $B$ in Tesla) (or $\chi_{\perp}$ equal to a fixed constant consistent with experimental observations) based on electrons and ions, respectively, for a hydrogen plasma at $n=5 \times 10^{19} \mathrm{~m}^{-3}$. When $\chi_{\perp}$ is computed from the evolving fields, the calculation is simplified by using toroidal averages (indicated by \langle\rangle , hereafter) of the evolving temperature and magnetic induction fields. Similarly, the electrical diffusivity is computed as $\eta / \mu_{0}$ $=411(1 \mathrm{eV} /\langle T\rangle)^{3 / 2} \mathrm{~m}^{2} / \mathrm{s}$. (Numerical tests show no significant deviation from results with a three-dimensional computation of resistivity in the conditions of interest. The results of earlier simulations with $0 \leqslant n \leqslant 2$ and a three-dimensional resistivity instead of a two-dimensional resistivity yielded a $1.5 \%$ difference in internal energy during a period of time when the internal energy increased by $15 \%$. We note that during formation when there are significant magnetic fluctuations, the field lines are chaotic and open, which results in relatively uniform temperature distributions because of efficient parallel heat conduction. As the confinement improves, flux surfaces emerge and the temperature peaks poloidally but remains relatively symmetric toroidally within the flux surfaces and within regions of confined or open chaotic field lines.) An isotropic viscosity $v$ of $100-1000 \mathrm{~m}^{2} / \mathrm{s}$ is used to provide nonlinear numerical stability during the full-power stage of the current drive. With temperatures of $\approx 30 \mathrm{eV}$ during this stage, the Lundquist number (computed as $S$ $=\mu_{0} R \mathrm{v}_{A} / \eta$, where $R$ is the radius of SSPX and $v_{A}$ is the Alfvén speed) is of order $10^{6}$. This is much larger than values considered in earlier spheromak simulations. ${ }^{3,4}$ The diffusion term in Eq. (1) keeps the density relatively smooth in the absence of particle transport and atomic fueling effects that are not present in the MHD model. The artificial diffusivity $D$ is set to $2000 \mathrm{~m}^{2} / \mathrm{s}$ to help keep the computed minimum of the number density field above zero during strong drive when the MHD activity is violent. For the same reason, the diffusivity is increased locally in computational cells where $n$ falls to $3 \%$ of its volume-averaged value. In future work we shall report simulation studies examining the effects of reducing $D$ to smaller values. We believe that the strong influence of parallel heat conduction is expected to dominate the role of $D$ in the artificial transport or loss of energy. Because the radiation power is small in most SSPX shots after proper wall conditioning, radiation is not modeled in the NIMROD simulations.

In conditions with sustained coaxial electrostatic drive, the cold edge plasma impedes parallel thermal conduction to the wall, despite the chaotic magnetic topology, allowing the plasma core temperature to reach tens of eVs. When the drive is temporarily removed, relatively symmetric closed flux surfaces form following the resistive decay of symmetry-breaking modes. Magnetic reconnection occurs rapidly in the cold outer plasma, and core temperatures increase toward $100 \mathrm{eV}$ or more. Applying a second current pulse (sustainment pulse), as in some SSPX discharges, ${ }^{2,6,7}$ is shown to improve performance by delaying the onset of MHD modes that are resonant in the closed-flux region, and higher current, increased magnetic fields, and larger volumes of closed flux can be achieved if the gun current is carefully chosen (discussed in Secs. II and III). The simulations reveal the sensitivity of the magnetic surfaces and the energy confinement with respect to symmetry-breaking magnetic fluctuations, and the close coupling of the magnetics and the energy transport. We have presented a detailed comparison of nonlinear simulations with laboratory measurements from SSPX (Ref. 2) and assessed confinement properties of the magnetic configuration in Ref. 8. Here we present comparisons of NIMROD simulation results to more recent SSPX discharges, and simulations motivated by discharges with repeated injector pulses. The simulation results are yielding electron temperatures, magnetic fields, and fluctuation amplitudes agreeing relatively well with SSPX observations.

The paper is organized as follows. Section II contains a description of simulations lam06 (with $\chi_{\perp}$ equal to a constant consistent with experiment) and lam07 (with $\chi_{\perp}$ de- 


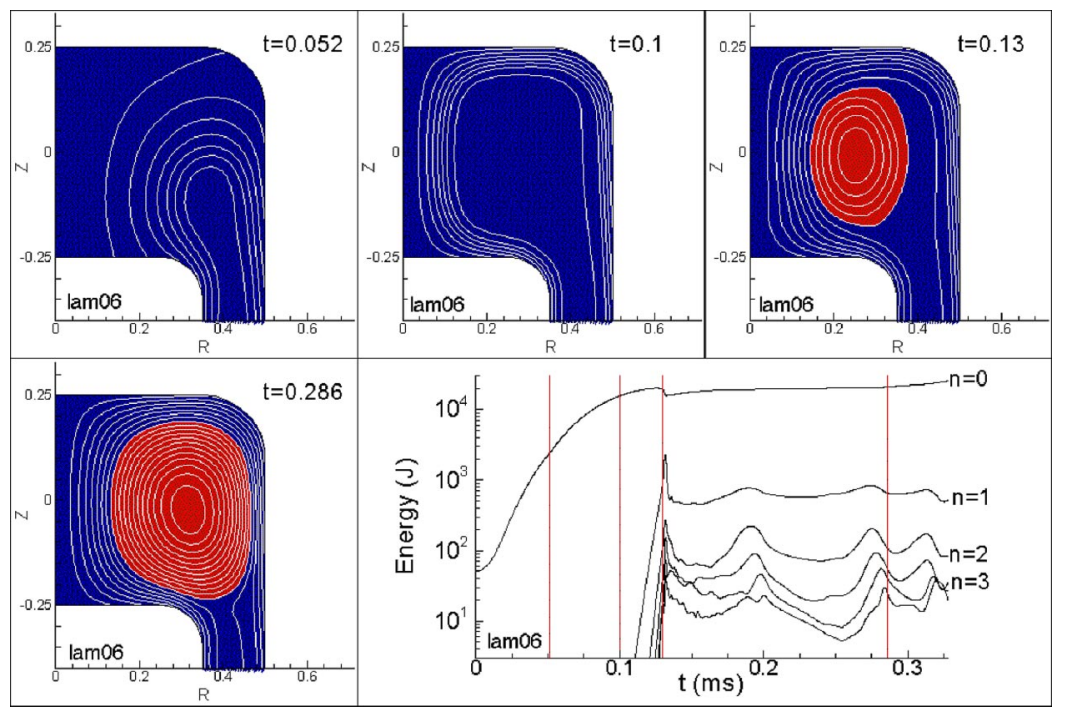

FIG. 2. Snapshots of the toroidally averaged poloidal magnetic flux showing spheromak formation and the magnetic energy as a function of time and toroidal Fourier mode number $n$ in NIMROD simulation lam06 with $\chi_{\perp}=21 \mathrm{~m}^{2} / \mathrm{s}$.

pending on temperature and magnetic field) directly modeling a series of SSPX shots driven by formation and sustainment pulses. The simulations agree well with many features in the SSPX data. In this section we also present examples of simulation results and diagnostics highlighting the physics of the magnetic reconnection that accompanies spheromak formation. We describe a few simulations addressing pulsed operation of a spheromak in Sec. III (simulations sphp09np, sphp09o, and sphp09p). We conclude with a brief summary and a discussion of future research directions in Sec. IV.

\section{SIMULATIONS OF FORMATION AND SUSTAINMENT PULSES IN SSPX}

The SSPX spheromak uses a coaxial plasma gun driven by a capacitor bank to drive current into the expanded region that is the flux conserver. ${ }^{2,6,7}$ A set of vacuum magnetic coils provides bias magnetic flux for the spheromak. Figure 1 shows a diagram of the spheromak, the vacuum magnetic field, and the time history of the gun injector current used in the simulations. These were constructed to model a series of shots in SSPX using formation and sustainment pulses. Reference 8 modeled SSPX shots 4620-4642. ${ }^{2}$ Here we have retained more of the elongated geometry of the plasma gun and modeled the detailed current-drive history corresponding to shots 12560 and 12705 .

In the NIMROD simulations magnetic flux is driven out of the plasma gun by $\mathbf{J} \times \mathbf{B}$ forces (shown for simulation lam06, for example, in Fig. 2 with $\chi_{\perp}=21 \mathrm{~m}^{2} / \mathrm{s}$ ) and reconnection occurs [abetted by the action of MHD instability with toroidal mode $n=1$ excited by the pinching of the current channel as seen routinely in SSPX in the formation stage and in CTX (Ref. 1) and the University of Manchester Spheromak experiment SPHEX (Ref. 9)]. In Fig. 3 we show comparisons of the injector current, gun voltage, and poloidal magnetic field measured near the midplane of the flux conserver by an edge probe in NIMROD simulation lam07 (using $\chi_{\perp}$ depending on the temperature and magnetic field) with measurements from SSPX shot 12705 during formation. Pulsations in the voltage are correlated with oscillations in the poloidal magnetic field history and reconnection events. ${ }^{10}$ With detailed field-line tracing using NIMROD
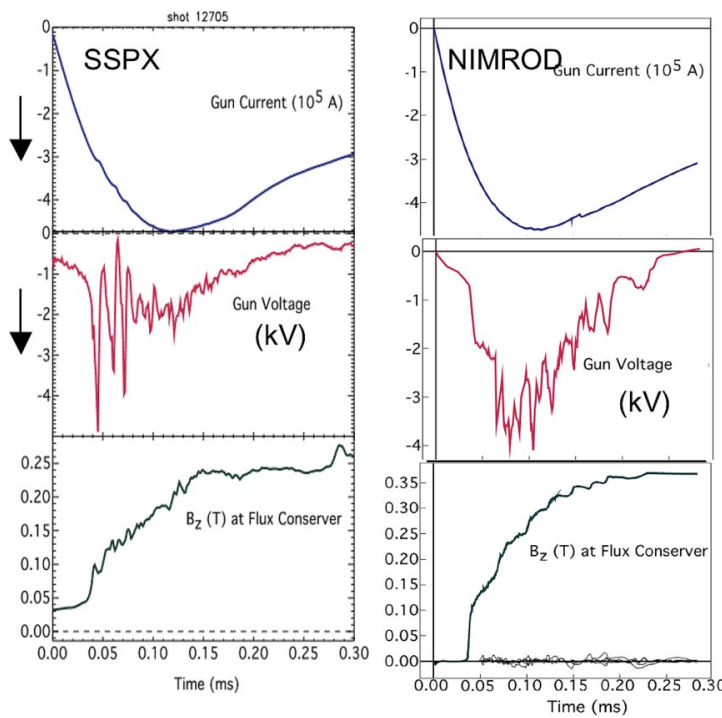

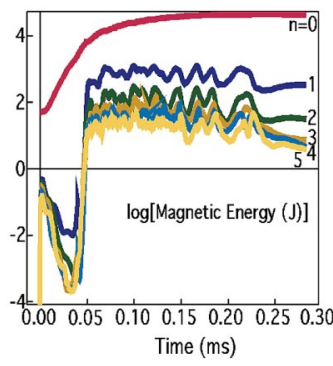

Time (ms)
FIG. 3. Injector current, gun voltage, and poloidal magnetic field measured at the midplane of the flux conserver by an edge probe in NIMROD simulation lam07 and measurements from SSPX shot 12705 during formation. Oscillations in the lam07 magnetic energy history correlate with oscillations in the voltage and $B_{z}(t)$. 


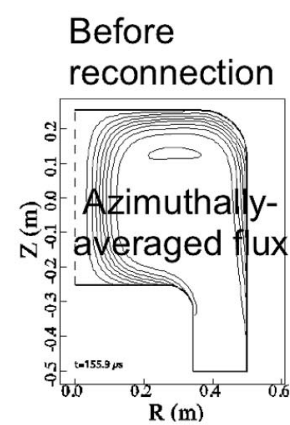

During and after reconnection

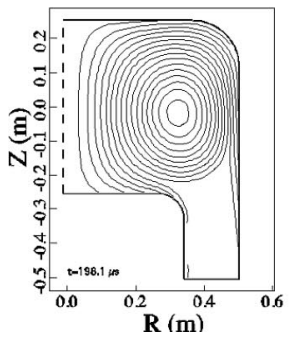

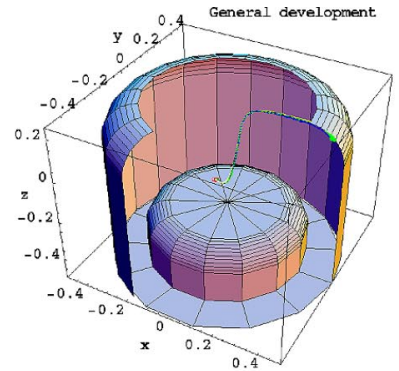

Field-line knotting

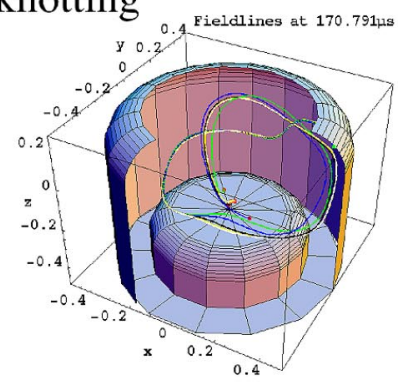

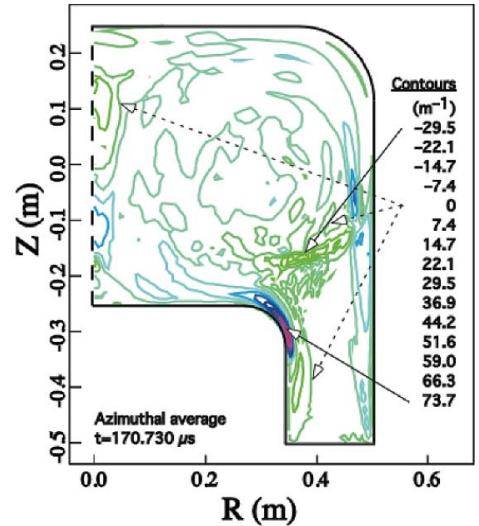

FIG. 4. Toroidally averaged poloidal flux, magnetic field lines, and $\lambda$ $=\mu_{0} \mathbf{J} \cdot \mathbf{B} / B^{2}$ contours showing topology change in the magnetic field attributed to reconnection in simulation lam07. Reconnection is generated by current sheets with negative $\lambda$, which are strongest near the $X$ point of the mean-field spheromak. simulation data, we see evolution of the magnetic topology and direct evidence of changes that we interpret as reconnection, e.g., formation of loose knots during formation (Fig. 4). This reconnection is accompanied by the appearance of current sheets with negative values of $\lambda=\mu_{0} \mathbf{J} \cdot \mathbf{B} / \mathrm{B}^{2}$ near the $X$ point of the toroidally averaged poloidal flux. A detailed examination of reconnection during spheromak formation is presented elsewhere. ${ }^{10}$

In Fig. 5 we compare time histories for the gun current, the poloidal magnetic field measured at an edge probe near the flux-conserver midplane, and peak electron temperatures from NIMROD simulation lam06 $\left(\chi_{\perp}=21 \mathrm{~m}^{2} / \mathrm{s}\right)$ and SSPX shot 12560 . The NIMROD simulation required nearly $10^{5}$ time steps and more than $2.5 \times 10^{4}$ processor hours extending over two months. The buildup in the poloidal magnetic field in the simulation tracked the experiment until $t$ $\sim 0.3 \mathrm{~ms}$ where there was an error in the current-drive model (the upward spike and downward correction in the simulated gun current in Fig. 5), which resulted in an accompanying spike and correction in the gun voltage and a steep decrease in the magnetic field. (The lam07 simulation has the smooth current drive history shown in Fig. 1 and will be featured in a future publication along with additional new work.) During the early part of the formation $(t<0.3 \mathrm{~ms})$, the lam06 simulation sees the $n=1$ toroidal mode at relative amplitudes similar to SSPX. The emergence of a current-driven $n=1$ mode during the formation of the spheromak with strong electrostatic drive is typical in both NIMROD simulations and experiments. ${ }^{1-9}$ After $0.5 \mathrm{~ms}$ the fluctuations begin to relax in the simulations and in the SSPX shot. At around $4 \mathrm{~ms} n=2$ fluctuations emerge in both the simulation and the experiment as the spheromak plasma dies. Figure 6 shows fluctuation data from SSPX discharge shot 12560 that is similar to that in lam06. Figure 5 also shows the time history of the peak electron temperature in lam06 and as measured by Thomson scattering in SSPX shot 12560 . With the gun
Shot 12560 NIMROD lam06

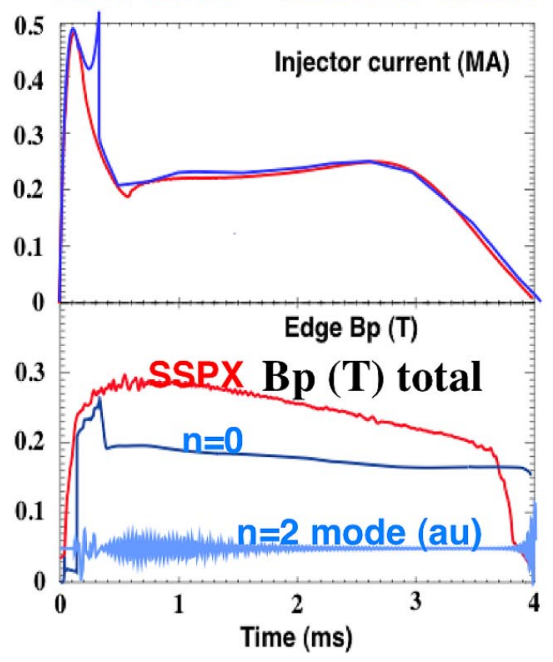

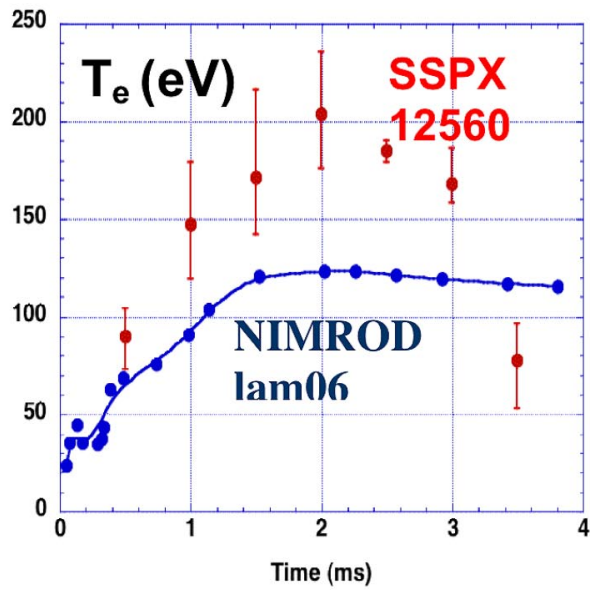

FIG. 5. Time histories of the edge poloidal magnetic field at the midplane of the flux conserver, the injector current, and the peak $T_{\mathrm{e}}$ in NIMROD simulation lam06 and SSPX shot 12560. 


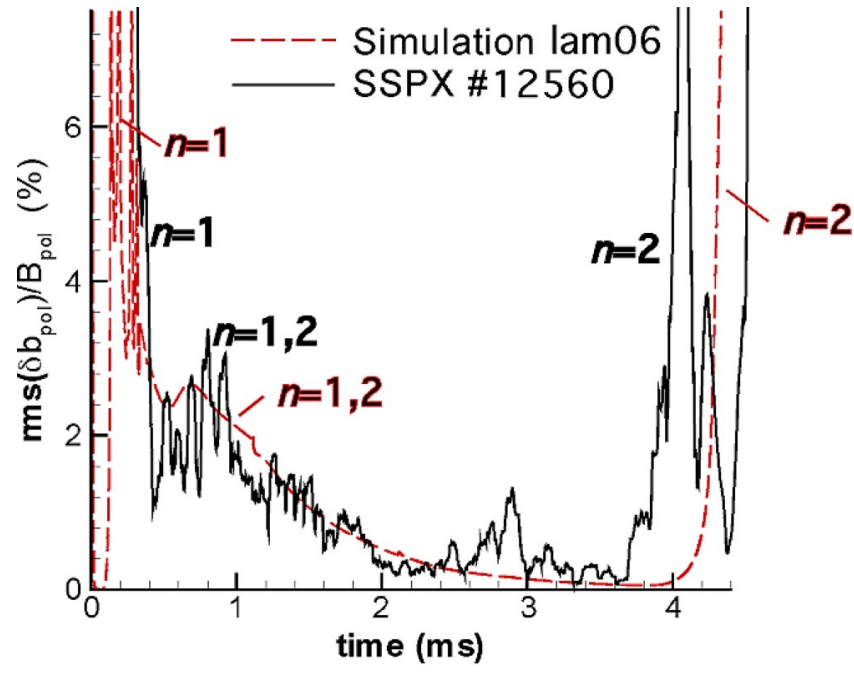

FIG. 6. Time histories of the edge-probe poloidal magnetic field fluctuation data from SSPX discharge shot 12560 and simulation lam06.

current reduced to a smaller value at $t \sim 0.3 \mathrm{~ms}$ in the lam06 simulation, the poloidal magnetic field and the plasma currents drop substantially below those in SSPX; the Joule heating rate decreases as a result (assuming that the resistivity is unchanged), which may account for $T_{\mathrm{e}}$ in lam06 falling below that observed in SSPX (pointed out by the referee). In addition, we note that in this NIMROD simulation we have used a single fluid model with $T_{\mathrm{e}}=T_{\mathrm{i}}$ assumed. If the ions were in fact colder than the electrons, this would imply that $T_{\mathrm{e}}$ might have to be higher to give the same total pressure as calculated in NIMROD. For the $120 \mathrm{eV}$ peak $T_{\mathrm{e}}$, the peak electron $\beta_{\mathrm{e}} \sim 4 \%$ in the simulation.

Figure 7 shows the poloidal magnetic topology (magnetic field-line puncture plot), corresponding $T_{\mathrm{e}}$ contours, and the spatial profile of $T_{\mathrm{e}}$ from SSPX shot 12560 . The temperature contours align with the magnetic field as a consequence of the parallel thermal conductivity being orders of magnitude larger than the perpendicular thermal conductivity. Magnetic islands link the temperature from one side of an island to the other and locally flatten the temperature profile. Closed, nested magnetic surfaces are surrounded by confined magnetic field lines that are chaotic, and the field lines are open surrounding the confined region. The transition from chaotic confined field lines to open field lines is accompanied by a steep drop in $T_{\mathrm{e}}$ which tracks the experimental Thomson scattering data well. Good energy confinement depends strongly on the presence of magnetic surfaces and the quality of the field lines. $T_{\mathrm{e}}$ of order tens of $\mathrm{eV}$ observed on the relatively short open magnetic field lines in the NIMROD simulations are in keeping with calculations in which Ohmic heating balances classical parallel energy transport. ${ }^{11,12}$

The MHD fluctuations tend to correlate with the presence of mode rational surfaces, and Taylor relaxation theory ${ }^{13}$ concludes that minimum energy states have flat $\lambda$ profiles. Thus, $\lambda$ gradients are a source of free energy to drive instability. Figure 8 shows plots of $\lambda$ and local safety factor $q$ at five times as functions of the poloidal flux $\langle\Psi\rangle$, where $q=d\langle\Phi\rangle / d\langle\Psi\rangle$, and $\langle\Phi\rangle$ and $\langle\Psi\rangle$ are toroidal and po-

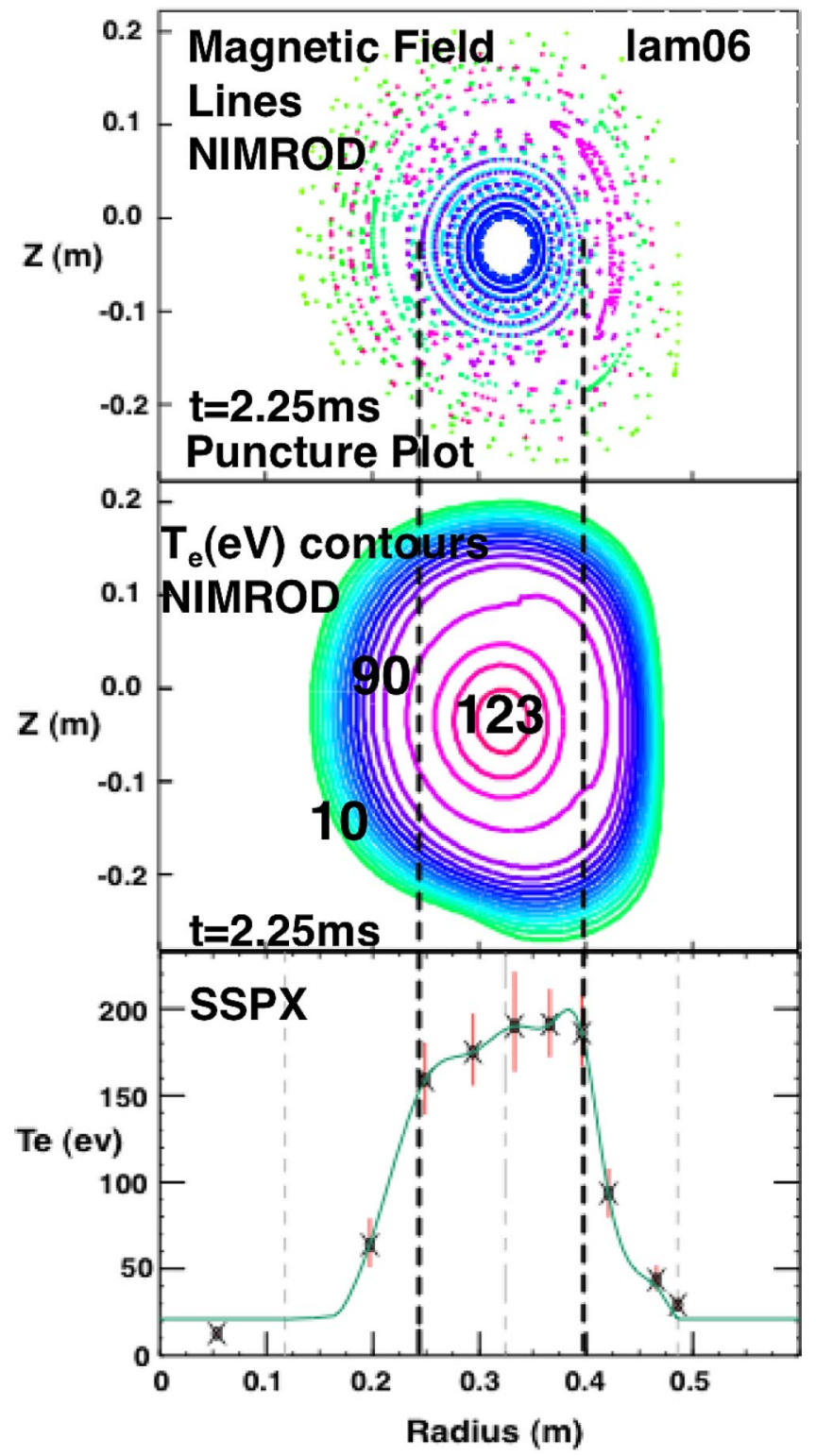

FIG. 7. Magnetic field-line puncture plot at a reference toroidal angle and the corresponding $T_{\mathrm{e}}$ contours from simulation lam06, and the spatial profile of $T_{\mathrm{e}}$ from SSPX shot 12560 .

loidal magnetic flux functions of $\langle\mathbf{B}\rangle$, respectively. In the region where the toroidally averaged poloidal magnetic flux is closed, we note that the $\lambda$ profile is relatively flat and approaches the eigenvalue $\lambda_{0}=9.6$ near the magnetic axis until late in time when a significant $\lambda$ gradient emerges accompanying the growth of an $n=2$ instability that is observed. We also note that the $q$ profile crosses from above $1 / 2$ to below going from $t=0.98 \mathrm{~ms}$ to $2 \mathrm{~ms}$. Mode rational surfaces, such as $q=2 / 3$ and $q=1 / 2$, evidently affect the MHD activity. Observations in SSPX and other NIMROD simulations show correlations between the $q$ profile crossing loworder mode rational surfaces and the emergence of fluctuations and islands. ${ }^{6-8}$ Magnetic islands with $m=2$ are visible in the lam06 simulation at $t=0.98 \mathrm{~ms}$ after the $q$ profile has crossed from above $2 / 3$ to below, inside the separatrix. Similarly, an $m=1$ island appears later in time after the $q$ profile 


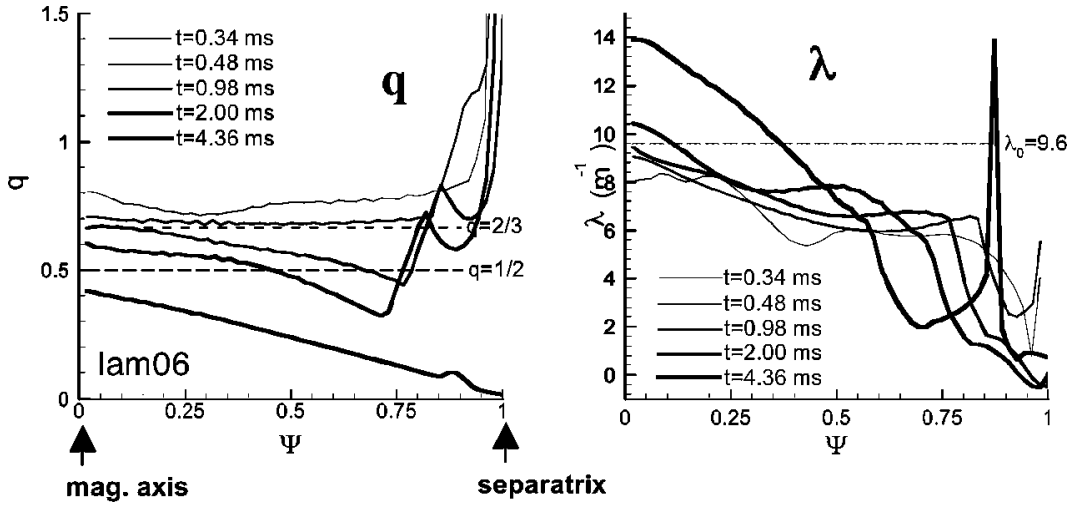

FIG. 8. Safety factor $q$ and $\lambda$ at five times as functions of the poloidal flux $\langle\Psi\rangle$ in NIMROD simulation lam06. has crossed $q=1 / 2$ inside the separatrix, and the $m=1, n$ $=2$ mode ultimately grows to significant amplitude (Fig. 6).

\section{SIMULATION OF PULSED SPHEROMAKS}

Spheromaks driven by coaxial plasma guns exhibit good confinement transiently. A strong formation pulse followed by a weaker sustaining pulse and decay allow magnetic fluctuations to relax and flux surfaces to emerge, with the highest temperatures appearing during sustainment and decay in experiments. ${ }^{1,2}$ The transient formation and decay behavior of spheromak plasmas is supported by simulations. ${ }^{3,4,8}$ With coaxial electrostatic current drive, symmetry-breaking fluctuations are needed to introduce a dynamo and provide helicity to maintain the spheromak against resistive decay. ${ }^{3,4,8}$ At the same time magnetic fluctuations can spoil the magnetic confinement, and previous simulations have shown that steady electrostatic drive leads to fluctuations that produce chaotic open field lines. ${ }^{3,4,8}$ If steady coaxial electrostatic current drive does not lead to good magnetic flux surfaces, can cyclically pulsed coaxial electrostatic current drive build high magnetic fields in a spheromak and produce good mag- netic surfaces cyclically? We have begun to use NIMROD simulations to address whether pulsed electrostatic drive can be optimized to achieve good energy confinement in a spheromak.

In Fig. 9 we display experimental results from SSPX shot 10233 in which there were two injected gun-current pulses. ${ }^{6}$ The second pulse in the experiment was able to build the magnetic fields to slightly higher levels in this example. We note that other SSPX shots have achieved significantly higher magnetic fields on the second pulse. ${ }^{6}$ The $T_{\mathrm{e}}$ response in time shows that local maxima occur during partial decay periods following each of the drive pulses. The NIMROD simulation (sphp09np) shown for comparison agrees qualitatively with SSPX in that the history of the peak $T_{\mathrm{e}}$ in the simulation shows local maxima in the two decay periods like the experiment and the values of $T_{\mathrm{e}}$ and the poloidal magnetic field in the simulation are similar to those in SSPX. This simulation did not attempt to mimic the experimental pulse shapes for the current drive (the pulse shapes in the simulation were simple $300 \mathrm{kA}$ square pulses, while the pulses in SSPX carried more peak current and were tapered

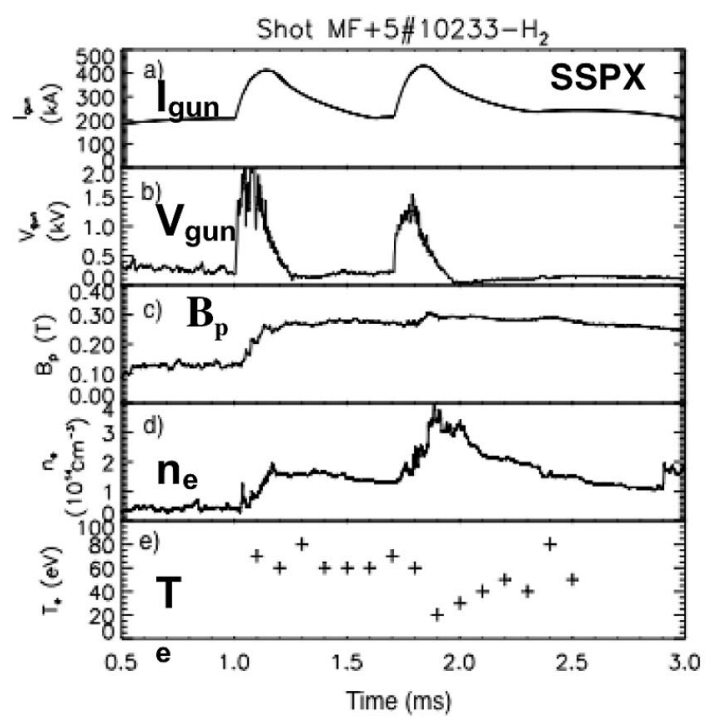

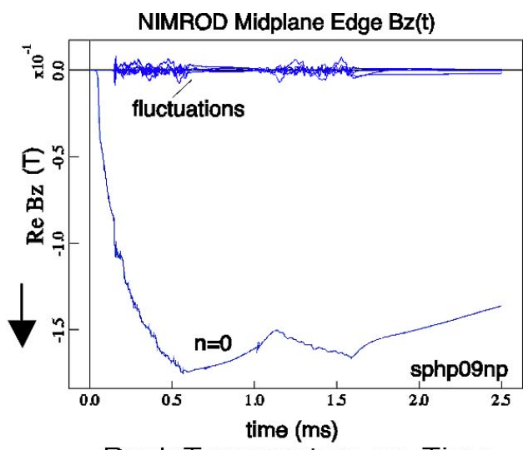

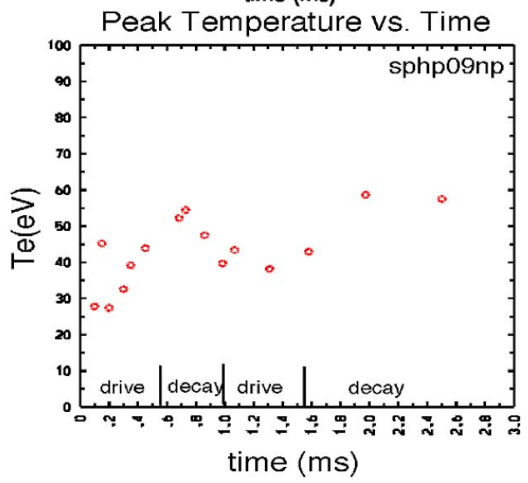

FIG. 9. (a) Time history data from SSPX shot 10233 and (b) time histories of the edge poloidal magnetic field near the flux-conserver midplane and the peak $T_{\mathrm{e}}$ in the NIMROD two-pulse simulation sphp09np. 

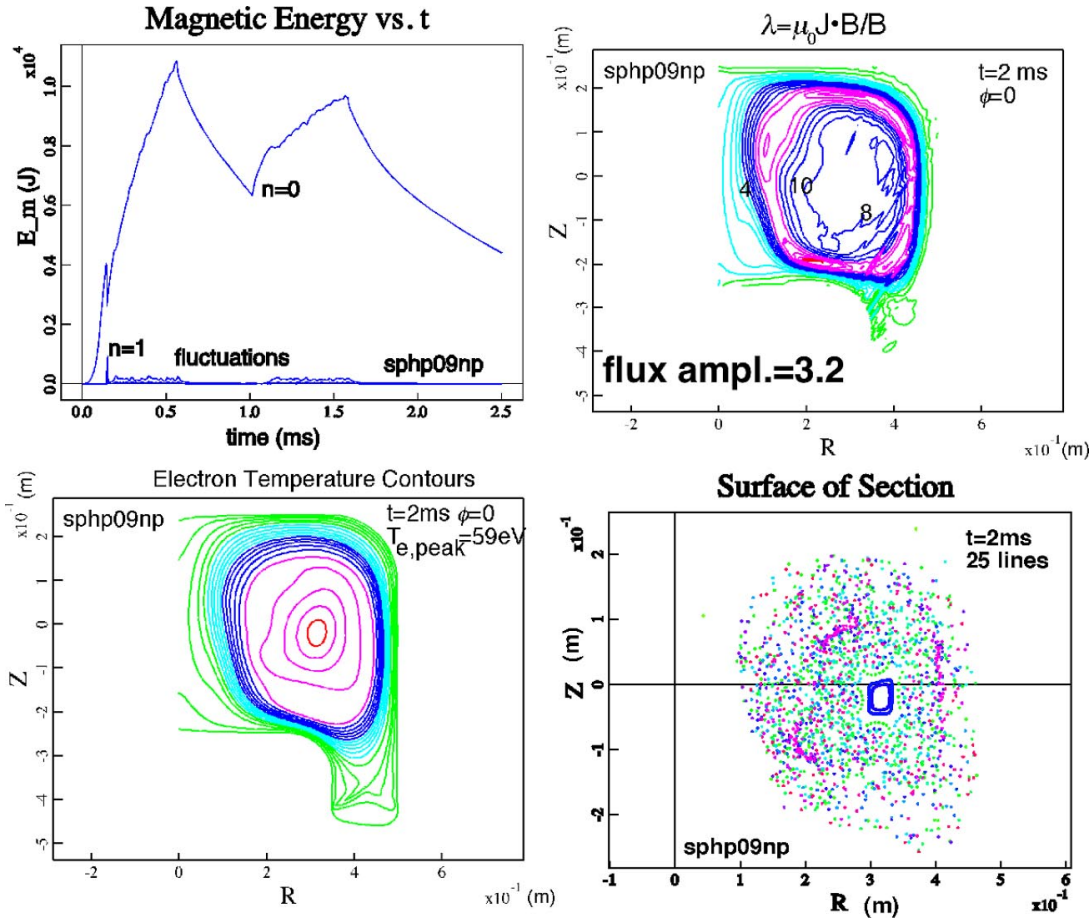

FIG. 10. Magnetic energy time history decomposed by toroidal Fourier mode, and at $t=2 \mathrm{~ms}: \lambda$ contours, $T_{\mathrm{e}}$ contours, and magnetic field-line puncture plot at a reference toroidal angle from the NIMROD two-pulse simulation sphp09np. in time); the magnetic field in the simulation on the second pulse did not grow to a higher value than in the first drive pulse. However, $T_{\mathrm{e}}$ in the second decay period in the simulation achieves a higher value than after the first pulse. The magnetic fluctuation amplitudes are higher during the strong drive periods and relax in the decay periods, during which time closed magnetic flux emerges near the magnetic axis surrounded by chaotic confined field lines (Fig. 10). The larger volume of closed, nested flux surfaces in the lam06 simulation shown earlier with tailored formation and sustainment pulses are accompanied by $50 \%$ higher peak injector currents than in sphp09np; the poloidal magnetic fields achieved are $\sim 25 \%$ larger; and the peak $T_{\mathrm{e}}$ is twice as large as those shown in Figs. 9 and 10. In both lam06 and sphp09np, the perpendicular thermal conductivity was $\chi_{\perp}$ $=21 \mathrm{~m}^{2} / \mathrm{s}$; we obtained results very similar to those in sphp09np when we used $\chi_{\perp}=15 \mathrm{~m}^{2} / \mathrm{s}$ in sphp09o (the minimum values observed in $\mathrm{SSPX}^{2}$ are $\chi_{\perp}=10-20 \mathrm{~m}^{2} / \mathrm{s}$ ).

In Fig. 11 we present the results of simulation sphp09p in which $\chi_{\perp}=15 \mathrm{~m}^{2} / \mathrm{s}$ and a single $300 \mathrm{kA}$, square currentdrive pulse of twice the duration $(\sim 1.2 \mathrm{~ms})$ as in sphp09np and sphp09o was used. The edge poloidal magnetic field shows evidence of saturation, and we note that the $L / R$ time for $T_{\mathrm{e}} \sim 20-35 \mathrm{eV}$ on open field lines is $\mu_{0} / \eta \lambda \sim 1 \mathrm{~ms}$ where the resistivity is $\eta \sim 10^{-5} \Omega \mathrm{m}$ here. The volume of closed, nested magnetic flux is slightly bigger in the singlepulse simulation than in the double-pulse simulation, and the peak $T_{\mathrm{e}}$ achieved during the decay is slightly higher. Overall the differences between the single and double-pulse simulations are small in terms of the spheromak plasmas obtained. The quality of the magnetic confinement is not outstanding in sphp09np, sphp09o, and sphp09p, and these plasmas are significantly colder than in the lam06 simulation. The shaped formation and sustainment pulses in the lam06 and lam07 simulations carefully model current-drive pulses used to ob- tain higher temperature discharges in SSPX. The sustainment pulses and the magnetic flux produced by the bias coils in SSPX and in lam06 and lam07 are constructed to provide a value of $\lambda_{\text {gun }} \equiv \mu_{0} I_{\text {gun }} / \Phi_{\text {gun }}$, where $I_{\text {gun }}$ is the gun injector current and $\Phi_{\text {gun }}$ is the bias magnetic flux at the gun throat, which approximately matches the $\lambda=\lambda_{0}=9.6 \mathrm{~m}^{-1}$ eigenvalue in the flux conserver. With $I_{\text {gun }} \sim 200 \mathrm{kA}\left(\lambda_{\text {gun }} \sim 8.4 \mathrm{~m}^{-1}\right)$ in the sustainment pulses following formation with higher current, plasmas with $T_{\mathrm{e}} \sim 100-200 \mathrm{eV}$ have been obtained in SSPX and NIMROD simulations. The $\lambda_{\text {gun }} \sim 12.6 \mathrm{~m}^{-1}$ used during the pulsed drive in the sphp09np and sphp09p simulations corresponds to overdriving the spheromak. Overdriving with $\lambda_{\text {gun }}$ significantly greater than $\lambda_{0}$ has not led to higher $T_{\mathrm{e}}$ in SSPX. We note that $I_{\text {gun }}=2.14 \times 10^{5} \mathrm{~A}$ in SSPX shot 10233 (Fig. 9) would correspond to $\lambda_{\text {gun }}=\lambda_{0}$, and thus SSPX shot 10233 was significantly overdriven at the peak of the two current pulses; only transiently was $\lambda_{\text {gun }} \approx \lambda_{0}$ satisfied, and $T_{\mathrm{e}}<85 \mathrm{eV}$ was observed. Experience in SSPX supported by NIMROD simulations suggests that matching $\lambda_{\text {gun }}$ and $\lambda_{0}$ during sustainment pulses reduces gradients in $\lambda$ that drive magnetic fluctuations, and reduced magnetic fluctuations lead to better flux surfaces and higher $T_{\mathrm{e}}$.

\section{CONCLUSION AND FUTURE DIRECTIONS}

The simulations presented here extend those in Ref. 8 in further elucidating the physics affecting spheromak formation and energy confinement, and showing improved agreement between NIMROD simulations and SSPX observations. The simulation results support SSPX observations in demonstrating that the relaxation of magnetic fluctuations after the spheromak is formed is key to obtaining better energy confinement and high temperatures, but leaves open the question of resupplying the helicity and energy to the spheromak core. The formation of magnetic islands as a conse- 

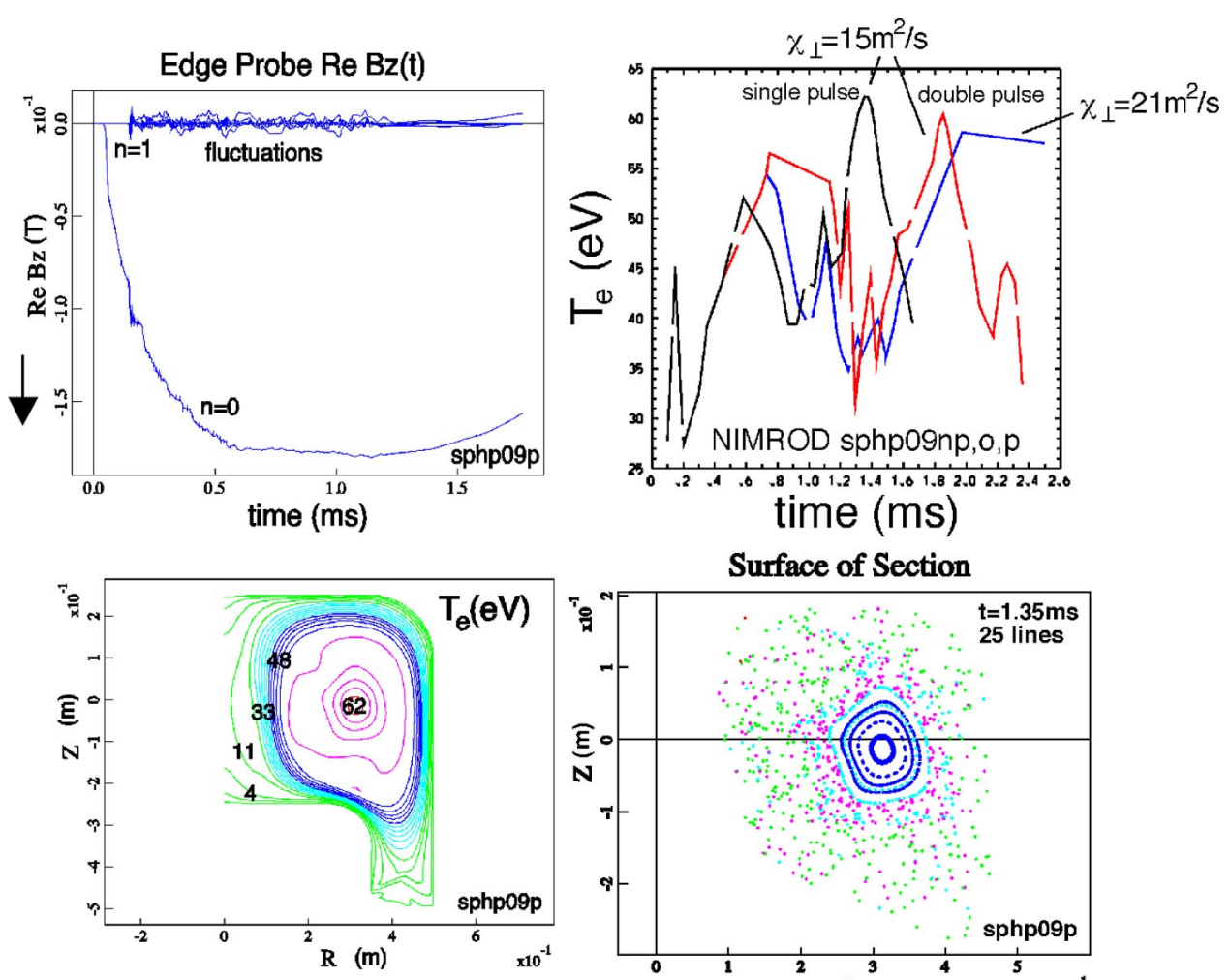

Surface of Section

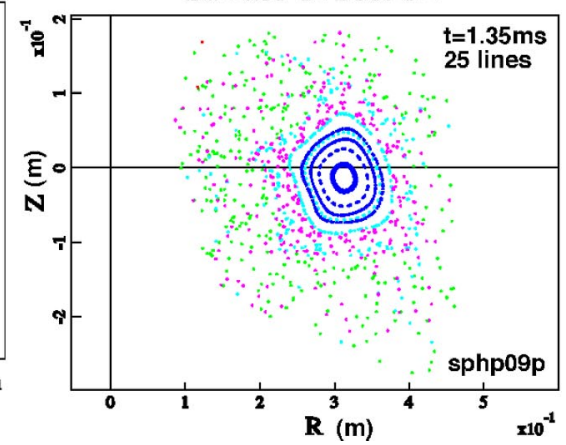

FIG. 11. Edge poloidal magnetic field time history near the midplane of the flux conserver in sphp09p; temperature time histories in NIMROD double-pulse simulations sphp09np $\left(\chi_{\perp}=21 \mathrm{~m}^{2} / \mathrm{s}\right)$ and $\operatorname{sphp09o}\left(\chi_{\perp}\right.$ $\left.=15 \mathrm{~m}^{2} / \mathrm{s}\right)$, and single-pulse simulation sphp09p $\left(\chi_{\perp}=15 \mathrm{~m}^{2} / \mathrm{s}\right) ; T_{\mathrm{e}}$ contours and magnetic field-line puncture plot at $t=1.35 \mathrm{~ms}$ in the single-pulse simulation sphp09p. quence of finite-amplitude magnetic fluctuations and the influence of the islands on energy transport are shown in the simulations. The quality of the magnetic field lines is critical in obtaining good energy confinement.

The simulations of pulsed operation of a spheromak are a work in progress. The contrast in the poorer magnetic surfaces and lower temperatures obtained in double-pulse simulations sphp09np $\left(\chi_{\perp}=21 \mathrm{~m}^{2} / \mathrm{s}\right)$ and $\operatorname{sphp09o}\left(\chi_{\perp}\right.$ $\left.=15 \mathrm{~m}^{2} / \mathrm{s}\right)$, and single-pulse simulation $\operatorname{sphp09p}\left(\chi_{\perp}\right.$ $=15 \mathrm{~m}^{2} / \mathrm{s}$ ) described in Sec. III with $\lambda_{\text {gun }}$ significantly larger than $\lambda_{0}$ (i.e., overdriven) compared to those in the lam06 and lam07 simulations with carefully tailored formation and sustainment pulses that try to match the current pulses used in SSPX indicates the importance of the detailed current-drive history and the principle derived from SSPX experience that matching $\lambda_{\text {gun }}$ to the $\lambda_{0}$ eigenvalue in the flux conserver leads to improved energy confinement. Future simulation work will include incorporating the beneficial effects of the sustainment pulses with $\lambda_{\text {gun }} \sim \lambda_{0}$ in controlling the $q$ profiles and reducing magnetic fluctuations in cyclically pulsed spheromak operation. Future simulations also will address the use of a conducting insert along the geometric axis to help control magnetic fluctuations, ${ }^{14}$ sensitivity studies with respect to the density diffusivity and the kinematic viscosity used to keep the numerics well behaved, and additional physics improvements to the simulation model.

The authors thank T. Kopriva for computing the magnetic field-line trajectories and producing the visualization of the field lines in Fig. 4. They are grateful to L. D. Pearlstein, T. K. Fowler, L. L. LoDestro, members of the SSPX experimental team at Livermore, and the NIMROD team for support, helpful discussions, and encouragement. They are also indebted to the referee for a very thorough review and making many valuable suggestions. For the simulations we made use of resources at the National Energy Research Supercomputer Center under U.S. Department of Energy Contract No. DE-AC03-76SF00098. This work was performed under the auspices of the U.S. Department of Energy under contract No. W7405-ENG-48 at the University of California, Lawrence Livermore National Laboratory, and Grant No. FG02-01ER54661 at the University of Wisconsin-Madison.

${ }^{1}$ T. R. Jarboe, Plasma Phys. Controlled Fusion 36, 945 (1994); T. R. Jarboe et al., Phys. Rev. Lett. 51, 39 (1983).

${ }^{2}$ H. S. McLean, S. Woodruff, E. B. Hooper, R. H. Bulmer, D. N. Hill, C. Holcomb, J. Moller, B. W. Stallard, and Z. Wang, Phys. Rev. Lett. 88, 125004 (2002); H. S. McLean, S. Woodruff, D. N. Hill et al., LLNL Laboratory Report No. UCRL-JC-152827 (unpublished); 30th EPS Conference on Controlled Fusion and Plasma Physics, Saint Petersburg, Russia, 7-11 July 2003; Europhys. Conf. Abstr. 27A, 3 (2003), http:// epsppd.epfl.ch/StPetersburg/PDF/P3_230.PDF

${ }^{3}$ C. R. Sovinec, J. M. Finn, and D. del-Castillo-Negrete, Phys. Plasmas 8, 475 (2001).

${ }^{4}$ R. H. Cohen, H. L. Berk, B. I. Cohen, T. K. Fowler, A. H. Glasser, E. B. Hooper, L. L. LoDestro, E. C. Morse, L. D. Pearlstein, T. D. Rognlien, D. D. Ryutov, C. R. Sovinec, and S. Woodruff, Nucl. Fusion 43, 1220 (2003).

${ }^{5}$ S. I. Braginskii, Reviews of Plasma Physics, edited by M. A. Leontovich (Consultants Bureau, New York, 1965), Vol. 1, p. 205.

${ }^{6}$ S. Woodruff, B. W. Stallard, H. S. McLean, E. B. Hooper, R. Bulmer, B. I. Cohen, D. N. Hill, C. T. Holcomb, J. Moller, and R. D. Wood, Phys. Rev. Lett. 93, 205002 (2004); S. Woodruff, B. I. Cohen, E. B. Hooper, H. S. McLean, B. W. Stallard, D. N. Hill, C. T. Holcomb, C. RomeroTalamas, R. D. Wood, G. Cone, and C. R. Sovinec, "Controlled and spontaneous magnetic field generation in a gun-driven spheromak," Phys. Plasmas (in press).

${ }^{7}$ S. Woodruff, D. N. Hill, E. B. Hooper, J. Moller, H. S. McLean, B. W. Stallard, R. D. Wood, R. Bulmer, and B. Cohen, Phys. Rev. Lett. 90, 95001 (2003). 
${ }^{8}$ C. R. Sovinec, B. I. Cohen, G. A. Cone, E. B. Hooper, and H. S. McLean, "Numerical Investigation of Transients in the SSPX Spheromak," Phys. Rev. Lett. 94, 035003 (2005)

${ }^{9}$ A. Al-Karkhy et al., Phys. Rev. Lett. 70, 1814 (1993).

${ }^{10}$ E. B. Hooper, T. A. Kopriva, B. I. Cohen, D. N. Hill, H. S. McLean, R. D. Wood, S. Woodruff, and C. R. Sovinec, "A magnetic reconnection event in a driven spheromak," Phys. Plasmas (submitted).
${ }^{11}$ E. B. Hooper, R. H. Cohen, and D. D. Ryutov, J. Nucl. Mater. 278, 104 (2000).

${ }^{12}$ R. W. Moses, R. A. Gerwin, and K. F. Schoenberg, Phys. Plasmas 8, 4839 (2001).

${ }^{13}$ J. B. Taylor, Phys. Rev. Lett. 33, 1139 (1974).

${ }^{14}$ D. D. Ryutov, R. H. Cohen, and L. D. Pearlstein, Phys. Plasmas 11, 4740 (2004). 